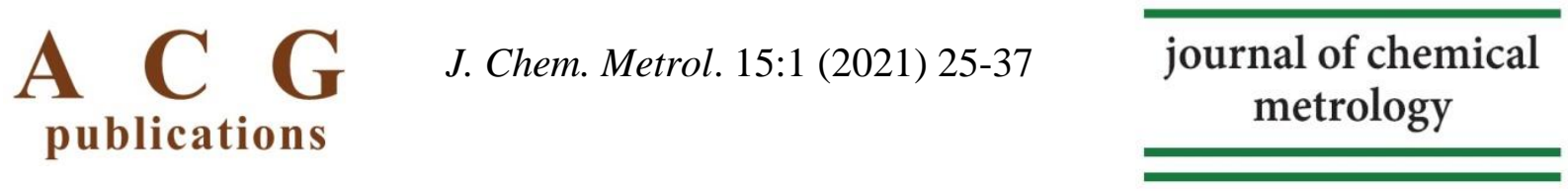

\title{
Uncertainty of small enthalpy effects measured by isothermal calorimetric titration
}

\author{
Astrid Darnell $\oplus^{1 *}$, Lauri Sikk $\oplus^{1}$, Ly Porosk $\oplus^{2}$ and Ivo Leito $\oplus^{1}$ \\ University of Tartu, Institute of Chemistry, Ravila 14A, Tartu, Estonia \\ University of Tartu, Institute of Technology, Nooruse 1, Tartu, Estonia
}

(Received March 02, 2021; Revised March 15, 2021; Accepted March 20, 2021)

\begin{abstract}
Combination of low concentration, low binding affinity and small solution volume is increasingly more common in calorimetric studies of host-guest interaction, but it leads to very small enthalpy and entropy effects, which are difficult to measure accurately. The aim of this study was to evaluate the reliability of enthalpy determination under such conditions using isothermal titration calorimetry (ITC) with a small-volume microcalorimeter and to evaluate the expected accuracy (expressed as measurement uncertainty) on the example of a simple 1:1 binding reaction of 18-crown-6-ether with $\mathrm{K}^{+}$cation. The investigation focused on whether or not it is possible to get meaningful results from experiments on such a system with low binding constant, low concentration $(\log K \approx 2)$, low Wiseman " $c$ " parameter values $(c<1)$ and low total heat released in the studied system. A thorough estimation of measurement uncertainty was performed using the component-by-component (the "classical" GUM) approach to estimate the uncertainties of experimentally determined heat effects at individual titration points and applying those uncertainties to data fitting to obtain the $K$ and $\Delta H$ values for the studied reaction. We found that it was possible to determine the reaction enthalpy $\Delta H$ of $-26.6 \mathrm{~kJ} / \mathrm{mol}$ with standard uncertainty of $1.1 \mathrm{~kJ} / \mathrm{mol}$. If we assume similar uncertainties for the other authors, the $\Delta H$ value found in this work is in good agreement with the majority of the literature $\Delta H$ values and those are in general in agreement with each other.
\end{abstract}

Keywords: Isothermal titration calorimetry; Wiseman $c$ parameter; measurement uncertainty; studying the binding of low affinity systems. () 2021 ACG Publications. All rights reserved.

\section{Introduction}

For assessing the achievable accuracy of calorimetric measurements, the Wiseman " $c$ " parameter [1] is commonly used as a criterion. This parameter ties together the cell reagent concentration in the calorimeter, the reaction stoichiometry and the equilibrium constant of the studied reaction. The Wiseman " $c$ " parameter is calculated using Equation (1), where $n$ is the reaction stoichiometry, $K$ the binding constant of the reaction and $M_{\text {tot }}$ the initial cell concentration of the used compound.

$$
c=n \times M_{\mathrm{tot}} \times K
$$

The shape of the titration curve obtained via calorimetric experiment depends on the c parameter value. Commonly, " $c$ " parameter values ranging between 5 and 500 are used for ITC experiment planning [2].

\footnotetext{
* Corresponding author E-Mail: astrid.darnell@ut.ee

The article was published by ACG Publications 
In a traditional ITC experiment around 25 injections of similar volume are made with a solution of the guest molecule/ion with concentration 10-20 times higher than the host molecule solution in the calorimetric cell. This results in a total amount of approx. 2 equivalents of titrant added by the end of the titration experiment [3]. The generally accepted position in ITC is that " $c$ " parameter values lower than 1-10 (different references quote different values) are considered unacceptable as the titration curve becomes flattened and loses certain notable characteristics $[1,2,4]$. This makes obtaining accurate results difficult or impossible [2].

Table 1. Experimentally determined $\log K$ and $\Delta H$ (in $\mathrm{kJ} / \mathrm{mol}$ ) values of binding $\mathrm{K}^{+}$by $18 \mathrm{C} 6$ in water at 298.15K from previous studies [7,9-15].

\begin{tabular}{|c|c|c|c|c|c|c|c|c|}
\hline \multirow{2}{*}{\multicolumn{2}{|c|}{ Source }} & \multicolumn{7}{|c|}{ Method information and experimental data from past publications } \\
\hline & & \multirow{2}{*}{$\begin{array}{c}\text { Method }^{\text {a }} \\
\text { IPC }\end{array}$} & \multirow{2}{*}{$\begin{array}{c}\text { Instrument } \\
\begin{array}{c}\text { isoperibol titration } \\
\text { calorimeter }\end{array}\end{array}$} & \multirow{2}{*}{$\frac{\log K}{2.03}$} & \multirow{2}{*}{$\begin{array}{c}\begin{array}{c}\text { Unc. } \\
(\log K)\end{array} \\
0.1\end{array}$} & \multirow{2}{*}{$\frac{\Delta \boldsymbol{H}^{\mathrm{b}}}{-25.98^{\mathrm{b}}}$} & \multirow{2}{*}{$\begin{array}{l}\text { Unc. } \\
(\Delta H)\end{array}$} & \multirow{2}{*}{$\begin{array}{c}\text { Unc. } \\
\text { type }\end{array}$} \\
\hline [9] & 1976 & & & & & & & \\
\hline [10] & 1982 & $\begin{array}{l}\text { Pot }(K) \\
\text { C }(\Delta H)\end{array}$ & LKB 8700 & $2.14^{\mathrm{c}}$ & $0.02^{\mathrm{c}}$ & $-23.43^{b}$ & $0.84^{\mathrm{b}}$ & $\mathrm{s} * \mathrm{t}_{\text {coeff }}$ \\
\hline [11] & 1987 & $\mathrm{C}$ & LKB $8721-2$ & 1.97 & 0.05 & -26.02 & 0.29 & $\pm \mathrm{x}$ \\
\hline [12] & 1992 & ITC $^{\mathrm{d}}$ & $\begin{array}{l}\text { "fully-automated online } \\
\text { calorimetry system" }\end{array}$ & 2.04 & 0.1 & -26.3 & 0.2 & $3 * \mathrm{~s}$ \\
\hline [13] & 1993 & IPC & $\begin{array}{c}\text { Tronac model } 450 \\
\text { isoperibol titration } \\
\text { calorimeter }\end{array}$ & $2.037^{\mathrm{e}}$ & $0.004^{\mathrm{e}}$ & -24 & 0.4 & $\pm \mathrm{x}$ \\
\hline [14] & 1995 & IFC & $\begin{array}{l}\text { isothermal flow } \\
\text { calorimeter }\end{array}$ & 2.06 & 0.02 & -26.0 & 0.1 & $\pm \mathrm{x}$ \\
\hline$[7]^{\mathrm{e}}$ & 2003 & ITC $^{\mathrm{f}}$ & VP-ITC & $\begin{array}{l}2.01 \ldots \\
2.1^{\mathrm{e}}\end{array}$ & $\begin{array}{l}0.01 \ldots \\
0.03^{\mathrm{e}}\end{array}$ & $\begin{array}{l}-28.8 \ldots \\
-32.9^{b}\end{array}$ & $\begin{array}{c}0.3 \ldots \\
1.2^{\mathrm{b}}\end{array}$ & $2 * s^{g}$ \\
\hline [15] & 2008 & ITC & $\begin{array}{l}\text { Tronac model } 450 \\
\text { isoperibol titration } \\
\text { calorimeter }\end{array}$ & 2.10 & 0.08 & -25.0 & 1.3 & $\pm \mathrm{x}$ \\
\hline
\end{tabular}

${ }^{a}$ IPC- isoperibol titration calorimetry; ITC - Isothermal titration calorimetry; Pot- potentiometry; C - Non-

isothermal or unspecified calorimetric experiment; IFC - Isothermal flow calorimetry.

${ }^{\mathrm{b}}$ Units converted from kcal mol${ }^{-1}$ to $\mathrm{kJ} \mathrm{mol}^{-1}$ via multiplying with $4.184 \mathrm{~J} / \mathrm{cal}$.

${ }^{\mathrm{c}}$ Estimated from $\Delta G$ values in the original publication.

${ }^{\mathrm{d}}$ Constant ionic medium, $0.1 \mathrm{M}\left(\mathrm{C}_{2} \mathrm{H}_{5}\right)_{4} \mathrm{NCl}$ added to the aqueous solution.

${ }^{\mathrm{e}}$ Converted from $K$ values in the original publication.

${ }^{\mathrm{f}}$ In this study, multiple determinations were carried out, varying the experimental concentrations.

${ }^{\mathrm{g}}$ For some other reactions studied in the paper; error estimates given by ORIGIN are also mentioned in this paper.

In supramolecular chemistry in general and in host-guest chemistry in particular the binding affinities towards the guest molecule or ion are often quite $\operatorname{low}(\log K<3)$ [5]. In addition, often there are limits to the solubility of the compounds, so that the experiments determining the thermodynamic parameters of binding will have to be carried out using low concentration of solutions. Planning the experiment to achieve both high " $c$ " parameter value and high rate of complexation to be reached at the end of titration is usually impossible due to needed guest molecule concentration being too high (insufficient solubility, inaccuracies in the measurement resulting from very large heat of dilution effects, very large heat effects at the start of titration dropping below instrumental power compensation level and rendering the peak area determination inaccurate etc.) [6]. As a result, in host-guest chemistry, calorimetry with suboptimal " $c$ " values (often below 1) is prevalent.

In the case of low affinity systems, the traditional experiment mentioned in the previous paragraph will not be ideal - with 2-3 equivalents of titrant added, only a fraction of the free receptor will be bound into complex by the end of the titration. At the same time, in order to apply the typically used Wiseman 
Darnell et al., J. Chem. Metrol. 15:1 (2021) 25-37

approach [1], the studied data should cover a sufficient range of the titration curve. Past studies have advised that it is not possible to obtain accurate estimates of the thermodynamic parameters of the binding reaction from experiments that do not result in at least 50\% complexation rate by the end of titration [7]. As an alternative to the traditional calorimetric experiment, suggestions have been voiced that experiments using a variable injection volume [3] or high receptor to ligand concentration difference (to achieve sufficient complexation rates by the end of the experiment) $[7,8]$ may be usable for low " $c$ " parameter experiments. However, the accuracy of such experiments still has limitations related to accuracy of the syringe volume delivery of small volumes, correct accounting for heat of dilution with high concentration of solution in the syringe, instrumental limits, choice of data treatment method etc. [8]. For low- $c$ systems, multiple studies also note a strong correlation between the estimates of reaction stoichiometry $n$ and reaction enthalpy $\Delta H$ values $[3,7,8]$. This is not a problem in cases where the binding stoichiometry is previously known or can be accurately determined through another method, however, it does limit the applications of ITC for systems where the binding stoichiometry is previously unknown.

The problems of accuracy in calorimetric experiments in situations of low binding affinity are evidenced by the discrepancies in enthalpy effects measured by different authors for the same reaction. As an example, Table 1 gives information on experimentally determined enthalpy and binding constant values for 18-crown-ether (18C6) binding reaction with $\mathrm{K}^{+}$cations.

Table 1 gives a summary of the results of previous studies and their experimental determination of the $K$ and $\Delta H$ values of binding $\mathrm{K}^{+}$by $18 \mathrm{C} 6$ in water. The previously published experimentally determined $K$ values are in reasonably good agreement, while the $\Delta H$ values differ markedly between sources. It can also be seen, that the measurement uncertainty (usually termed "error" by authors) estimates for $\Delta H$ values are generally low (in the range of $0.04 . .0 .4 \mathrm{~kJ} / \mathrm{mol}$ ) and are by far insufficient to account for the large differences between the values. This is not surprising, as they are commonly estimated as standard deviation of the results from replicate determinations and therefore fail to account for the (often large) systematic effects.

Another illustration is given by a 2003 study that investigates how low " $c$ " parameter values can still be used for acceptable determination of $K$ and $\Delta H$ values, by studying $\mathrm{K}^{+}$and $\mathrm{Ba}^{2+}$ binding with $18 \mathrm{C} 6$. The resulting variance in the $\Delta H$ estimate for the $18 \mathrm{C} 6$ and $\mathrm{K}^{+}$binding reaction with varying the " $c$ " parameter from this study [7] is displayed in Table 2. Again, one can notice that the $\Delta H$ values differ quite strongly and the discrepancies between them are larger than can be justified by the experimental uncertainties, identified as standard deviations.

Table 2. Comparison of $\mathrm{K}^{+}$and $18 \mathrm{C} 6$ binding reaction equilibrium constant $(K)$ and enthalpy $(\Delta H)$ estimates and experimental " $c$ " parameter from a 2003 study [7]. In the study, different $c$ values were obtained through varying the experimental concentrations.

\begin{tabular}{|c|c|c|}
\hline \multirow{2}{*}{$c$ parameter } & \multicolumn{2}{|c|}{ Determination of $K$ and $\Delta H$ estimates at various $c$ parameter values [7] } \\
\hline & $K$ & $\Delta H(\mathrm{~kJ} / \mathrm{mol})^{a}$ \\
\hline 10 & $\begin{array}{c}117 \pm \\
6\end{array}$ & $-32.9 \pm 0.4$ \\
\hline 5 & $\begin{array}{c}104 \pm \\
2\end{array}$ & $-32.7 \pm 0.3$ \\
\hline 1 & $\begin{array}{c}119 \pm \\
4\end{array}$ & $-31.8 \pm 0.3$ \\
\hline 0.1 & $\begin{array}{c}110 \pm \\
2\end{array}$ & $-30.1 \pm 0.6$ \\
\hline 0.05 & $\begin{array}{c}111 \pm \\
2\end{array}$ & $-30.5 \pm 0.3$ \\
\hline 0.01 & $\begin{array}{c}117 \pm \\
8\end{array}$ & $-28.8 \pm 1.2$ \\
\hline
\end{tabular}

${ }^{a}$ The values were converted from $\mathrm{kcal} \mathrm{mol}^{-1}$ used in the original publication to $\mathrm{kJ} \mathrm{mol}^{-1}$ via multiplying with 4.184 $\mathrm{J} / \mathrm{cal}$. The uncertainty estimates correspond to twice the standard deviations of three replicate measurements. 
The problem of mismatch between results from different authors could be related to using different models for calorimetric data treatment, problems with experimental setups or to lower instrumental accuracy of the older calorimeters used in some past studies $[2,8,16]$. However, it is also possible, that a large part of the discrepancy is the result of underestimating the measurement uncertainties. The uncertainty effects related to heat of dilution corrections, cell active volume and delivery of titrant volume can cause notable measurement uncertainty, but they are not always accounted for or may be underestimated. Obviously, all these uncertainty sources should be accounted for in the combined uncertainty estimate of the result.

Calorimetric experiments are very sensitive to certain experimental factors - determination of accurate concentration (especially that of the receptor in the cell) [7], vibration [16], and experimental baseline determination [16,17]. Studying $\mathrm{Ba}^{2+}$ and 18C6 binding, a 1999 study determined that enthalpy difference of up to $2 \mathrm{~kJ} / \mathrm{mol}$ may arise from the use of different calorimeters [18].

Often, the uncertainty of the determined enthalpy values is estimated through standard deviation of results of replicate determinations $[7,9,10,15]$. Some of the commonly used data treatment programs (e.g. ORIGIN), also give an uncertainty estimate for the determined $n, K$ and $\Delta H$ values, however, this estimate tends to be very small by its magnitude and typically only estimates the goodness-of-fit between experimental and theoretical titration curves[7]. The use of standard deviation or goodness-of-fit as an estimate for overall measurement uncertainty does not include other possible sources of uncertainty (especially those that have systematic effects) or, in some cases, may underestimate them.

Previously, some studies have been conducted specifically investigating the main uncertainty sources related to measurement and statistical data treatment of ITC experiments $[16,17,19]$. In these studies, the measurement uncertainty components (often termed "error" in by authors) of calorimetric measurements are estimated through the component-by-component approach realized through MonteCarlo methods [16].

Some past studies specifically focus on investigating the possibility of accurate calorimetric measurements for low " $c$ " conditions. The studies reach the general conclusion that with careful experiment planning and avoiding errors in data treatment, results may be obtained for calorimetric experiments carried out under low " $c$ " parameter conditions $(c<1)$ [6,7]. While several studies focus on estimating contributions of specific sources of measurement uncertainty, these estimations generally focus on the contribution of specific uncertainty sources that may affect the result of the measurement (e.g. a study focusing on investigation the effects of concentration uncertainty may not carry out a full estimation of volume delivery related uncertainty contributions etc.) and do not present a full uncertainty budget of the contributions of different sources of uncertainty.

The aim of this study was to thoroughly investigate the contribution of all relevant uncertainty sources in such low " $c$ " measurements carried out with a small-volume microcalorimeter, to the estimate of measurement uncertainty for determining the $K$ and $\Delta H$ values and estimate the level of accuracy for $\Delta H$ determination at low " $c$ " parameter conditions.

For uncertainty estimation in this work, the following steps were taken:

- The model reaction for this study was chosen by the following criteria - low binding affinity, availability of extensive experimental data from previous studies, known 1:1 binding stoichiometry of the reaction, commercial availability of reagents in suitable purity. The binding reaction of 18 -crown- 6 ether and $\mathrm{K}^{+}$cation matches all these criteria and was used for this study.

- The sources of measurement uncertainty were identified. All steps of the analysis were considered for their potential contribution to the measurement uncertainty - from experiment planning, the instrumental measurement to data fitting.

- The study investigated the uncertainty contributions of active volume estimation, volume delivery by injections during the measurement, the subtraction of heat of dilution effects of adding the cation solution into the solution in the calorimetric cell, suitable mathematical model selection to best describe the calorimetric experiment and suitable choice of fit model in the data treatment.

- Where possible, the uncertainty contributions of the uncertainty sources were quantified experimentally. 
Darnell et al., J. Chem. Metrol. 15:1 (2021) 25-37

- The $\Delta H$ and $K$ values were determined from the experimental data together with uncertainty estimates using a Python script.

- The component-by-component approach was used to estimate the uncertainty of the obtained reaction enthalpy values.

\section{Experimental}

\subsection{Materials and Methods}

18-crown-6-ether was purchased from Arcos Organics with 99\% purity. The $\mathrm{KCl}$ salt was purchased from Alfa-Aesar (99.95\% purity). A KF titration experiment in methanol was carried out to determine the water content in the crown-ether compound.

\subsection{ITC Experiments}

The ITC measurements were carried out with a MicroCal iTC unit at the UT Institute of Technology at $298 \mathrm{~K} .260 \mu \mathrm{L}$ of 18-crown-6-ether solution with the concentration of $5 \mathrm{mM}$ of was loaded into the calorimetric cell (active cell volume $200.6 \mu \mathrm{L}$ ) and salt solutions of 50 to $100 \mathrm{mM}$ were added in 19 steps of $2 \mu \mathrm{L}$ during the titration. An initial injection of $0.2 \mu \mathrm{l}$ was also made to account for the effects of diffusion of titrant into the cell during initial thermal equilibration and the corresponding data point was later removed from data analysis.

For all measurements, additional experiments were carried out to determine the effects of heat of dilution. The measured heat effects of these experiments were subtracted from the crown ether-cation titration curve before data analysis. All experimental results of this study are obtained from the data from which the heats of dilution have been subtracted from.

Four replicate experiments were carried out in a 2-month time period, varying the $\mathrm{K}^{+}$and $18 \mathrm{C} 6$ concentrations.

\subsection{Data Treatment}

A Python script was used (using the Nelder-Mead method [20] for finding the $\Delta H$ and $\log K$ values together with estimates of uncertainty for the goodness-of-fit.). For all experiments, the first data point was omitted to account for the effects of diffusion of titrant solution into the cell during the initial thermal equilibration process. For experiments $\mathrm{K} 3$ and $\mathrm{K} 4$, the first 5 peak areas were omitted from fitting due to inaccuracy of peak area determination due to too large heat effect values for the Microcal ITC $_{200}$ system. Examples of each curve fit option are shown in the SI.

\subsection{Instrument Calibration and Test Experiments}

The modern small-volume calorimetric instruments commonly used in modern laboratories for ITC ( ITC $_{200}$, PEAQ-ITC etc.) have relatively small used volumes (active cell volume approx. $200 \mu \mathrm{L}$, usable syringe volume up to $40 \mu \mathrm{L}$ ). While the manufacturer's manual advises that the injected volume accuracy should be $<1 \%$ for volumes as low as $2 \mu \mathrm{L}$ according to the manufacturer specifications, the accuracy of the instrumental volume delivery may decrease over time if instrument is not in an ideal working condition or if smaller volume delivery is needed. The following experiments were carried out to test the good working order of the $\mathrm{ITC}_{200}$ instrument:

- EDTA titration with $\mathrm{CaCl}_{2}$ in fixed $\mathrm{pH}$ MES buffer

- Pulse control experiment suggested by the manufacturer

- MQ to MQ control measurements

\subsection{Estimation of Standard Uncertainties for the Input Parameters of the Mathematical Model}

The stock solutions were made with MilliQ water into $15 \mathrm{ml}$ vials and the amounts of added substances determined by weighing with a 5-digit analytical balance Sartorius CPA225D-0CE. Working 
solutions were prepared from the standard solutions with pipetting the stock solutions and freshly taken MilliQ water into $15 \mathrm{ml}$ vials. All work was done at $23 \pm 3{ }^{\circ} \mathrm{C}$.

The standard uncertainty of solution concentrations (preparing stock solutions, dilution of stock solutions, transfer, stability) was, because of very small volumes involved, conservatively estimated at maximum $2 \%$.

During the similar-volume calorimetric experiment, 19 injections of $2 \mu \mathrm{L}$ were used. The standard uncertainty in volume for each injection was estimated at $2 \%$.

The standard uncertainty of the cell fill level was estimated at $4 \mu \mathrm{L}$, which was used as an estimate of the uncertainty of the active cell volume. The active volume of the cell is $200.6 \mu \mathrm{L}$.

\section{Results and discussion}

\subsection{Uncertainty Estimation: Overview}

While the overall goal of this study is to estimate the measurement uncertainty of reaction enthalpy $(\Delta H)$, it is not possible to estimate this uncertainty directly from the titration data. The enthalpy estimate is found (together with the equilibrium constant $K$ ) by least squares fitting a model to the experimental titration curve, composed of the values of the heat effects corresponding to the individual titrant injections made during titration. Thus, in order to obtain information about the total heat released by the system, the approach to uncertainty estimation initially centers on the determination of heat effects (peak areas) corresponding to individual titrant injections and focuses on estimating the measurement uncertainties for these heat effects realized using the component-by-component approach. As the next step, the least squares fitting was carried out for finding the reaction $\Delta H$ and $K$ values, as well the estimate of uncertainty for the fit. However, this estimate only considers the goodness of fit of the model with the experimental points and misses systematic effects that affect the whole curve. Thus, it cannot be used to estimate the overall uncertainty of the determined enthalpy values. For the uncertainty estimation of the enthalpy of the binding reaction, the component-by-component approach is used to estimate the uncertainty from the mathematical model that is also used to estimate the heat effects of binding for calorimetric experiments (see the following paragraph).

\subsection{Uncertainty Estimation of Experimentally Determined Heat Effects of Individual Injections}

The estimation of measurement uncertainty for heat effects of the experimental titration points is carried out using these steps [21]:

-A suitable model is chosen (described below) for calculating the measured heat effects $\Delta Q(\mathrm{i})$ of individual injections in the calorimetric experiment.

-The sources of measurement uncertainty are identified and linked to the input quantities in the model.

-The uncertainty components related to the input quantities are quantified using the Kragten approach.

-The components are combined to estimate the uncertainties of the measured values of the heat effects of individual injections.

For choosing the mathematical model that best describes the system and the experiment, it is important that $\mathrm{ITC}_{200}$ is a perfusion instrument, where the calorimetric cell has a certain active volume and an overflow compartment above it. In the preparation of a calorimetric experiment, the cell is overfilled with a larger solution volume than $V_{0}$ to avoid air bubble formation inside the calorimetric cell, then the excess volume is removed before inserting the calorimeter syringe. During measurement the instrument can directly "see" only the active volume of solution during all the measurements and only measures the heat effects of reactions happening in this active volume. With every injection some of the solution is pushed into the overflow compartment. However, the reaction takes place also in the overflow compartment and its heat effect could thus slightly affect the measured equilibrium/heat. During each injection, the injected volume of titrant is added to the calorimetric cell active volume, and an equal volume of the solution that is currently in the active volume is pushed out from the active volume. 
The heat effect of binding is expressed via the following equation [22]:

$$
Q=\frac{n M_{\mathrm{t}} \Delta H V_{0}}{2}\left[1+\frac{X_{\mathrm{t}}}{n M_{\mathrm{t}}}+\frac{1}{n K M_{\mathrm{t}}}-\sqrt{\left(1+\frac{X_{\mathrm{t}}}{n M_{\mathrm{t}}}+\frac{1}{n K M_{\mathrm{t}}}\right)^{2}-\frac{4 X_{\mathrm{t}}}{n M_{\mathrm{t}}}}\right]
$$

Here, $Q$ describes the total (cumulative) measurable heat effect of the process in the solution in the active volume of the calorimetric cell, $\mathrm{n}$ is the stoichiometry coefficient of the reaction, $M_{\mathrm{t}}$ is the bulk concentration of the receptor, $\Delta H$ the molar enthalpy effect of the binding reaction, $V_{0}$ is the active volume of calorimeter, $X_{\mathrm{t}}$ is the bulk concentration of the cation, and $K$ is the equilibrium constant.

The calculations of bulk concentrations for the receptor and cation in the calorimetric cell take into account the active volume corrections, according to equations (3) to (5) [22] :

$$
\begin{aligned}
& M_{\mathrm{t}}=M_{\mathrm{t} 0}\left[\frac{1-\frac{V \text { tot }_{\mathrm{i}}}{2 V_{0}}}{1+\frac{V \text { tot }_{\mathrm{i}}}{2 V_{0}}}\right] \\
& X_{\mathrm{t}}=X_{\mathrm{t} 0}\left[\frac{1}{1+\frac{V \mathrm{tot}_{\mathrm{i}}}{2 V_{0}}}\right] \\
& X_{\mathrm{t} 0}=c_{\text {syr }}\left[\frac{V \text { tot }_{\mathrm{i}}}{V_{0}}\right]
\end{aligned}
$$

Here, $M_{\mathrm{t} 0}$ and $X_{\mathrm{t} 0}$ are respective bulk concentrations before accounting for the volume effect. $M_{\mathrm{t} 0}$ is the initial $18 \mathrm{C} 6$ solution concentration in the cell, $c_{\mathrm{syr}}$ is the concentration of $\mathrm{K}^{+}$in the syringe, $X t_{0}$ is calculated by equation (5), where $c_{\mathrm{syr}}$ is the concentration of titrant solution in the calorimeter`s syringe, $V_{\text {tot, }}$ is the total injection volume after the i-th injection and $V_{0}$ the active volume of the calorimetric cell.

For estimating the heat released from i-th injection, $\Delta Q(\mathrm{i})$, the following equation is used [22]:

$$
\Delta Q(\mathrm{i})=Q(\mathrm{i})+\frac{v_{\mathrm{i}}}{V_{0}}\left[\frac{Q(\mathrm{i})+Q(\mathrm{i}-1)}{2}\right]-Q(\mathrm{i}-1)
$$

$Q(\mathrm{i})$ and $Q(\mathrm{i}-1)$ are the cumulative heat effects after the titrant injections i and i-1, respectively, both found from eq (2). The parameter $v_{\mathrm{i}}$ is the injection volume of the $\mathrm{i}$-th injection. The second term of the equation is used to account for the effect of displacing some of the solution from the active volume into the overflow compartment.

Equation (6) is used for obtaining the heat effects of individual injections that are used in the data fitting and also for the estimation of measurement uncertainties of these heat effects. An example of results for the estimation of measurement uncertainties for the heat effects of the individual injections and the importance of various uncertainty components during different parts of the titration experiment are discussed in the Supporting Information.

\subsection{The Overall Cumulative Heat effect of the Titration and Its Uncertainty}

The total cumulative heat effect of the entire ITC experiment, $Q_{\mathrm{tot}}$, is found by taking the sum of all heat effects of the titration peaks determined using equation (2) and is $Q_{\mathrm{tot}}=-7.54 \mathrm{~J}$, with standard uncertainty $u\left(Q_{\mathrm{tot}}\right)=0.18 \mathrm{~J}$ (on the example of experiment K1).

The uncertainty budget of $Q_{\mathrm{tot}}$ is displayed in Table 3. This uncertainty budget indicates that when uncertainty estimation is carried out for the heat released throughout the entire experiment, rather than individual injections, the active volume remains an important contributor to the measurement uncertainty, but the solution concentrations will have larger contributions towards the overall uncertainty. They are 
among the top contributors to the uncertainties of the first injections of the experiments, the heat effects of which are by far the largest.

Table 3. Uncertainty components of the input parameters for the determination of experimental heat effects. The uncertainty budget is given for the total amount of heat measured during the calorimetric experiment, $Q_{\mathrm{tot}}$.

\begin{tabular}{|c|c|c|c|c|c|}
\hline \multirow[b]{2}{*}{ Input parameters } & \multicolumn{5}{|c|}{ Uncertainty budget for $Q_{\text {tot }}$} \\
\hline & $\begin{array}{c}\text { Causes of } \\
\text { uncertainty }\end{array}$ & Estimated by & $\begin{array}{c}\text { Example } \\
\text { value }\end{array}$ & $\begin{array}{c}\text { Standard } \\
\text { uncertainty }\end{array}$ & $\begin{array}{l}\text { Contribution to } \\
Q_{\text {tot }} \text { uncertainty }\end{array}$ \\
\hline $\begin{array}{l}\text { Concentration of } \mathrm{K}^{+} \\
\text {in the syringe } c_{\mathrm{syr}}\end{array}$ & $\begin{array}{l}\text { reagent purity } \\
\text { solution creation }\end{array}$ & $\begin{array}{l}\text { component by } \\
\text { component } \\
\text { approach }\end{array}$ & $0.05 \mathrm{M}$ & $0.001 \mathrm{M}$ & $31 \%$ \\
\hline $\begin{array}{l}\text { Concentration of } \\
18 \mathrm{C} 6 \text { in the cell } M_{\mathrm{t} 0}\end{array}$ & $\begin{array}{l}\text { reagent purity } \\
\text { solution creation }\end{array}$ & $\begin{array}{l}\text { component by } \\
\text { component } \\
\text { approach }\end{array}$ & $0.005 \mathrm{M}$ & $0.0001 \mathrm{M}$ & $50 \%$ \\
\hline $\begin{array}{l}\text { Active cell volume } \\
V_{0}\end{array}$ & $\begin{array}{c}\text { precision of } \\
\text { manufacturer } \\
\text { estimate }\end{array}$ & $\begin{array}{c}\text { measurements } \\
\text { of fill level } \\
\text { volume }\end{array}$ & $200.6 \mu \mathrm{L}$ & $4 \mu \mathrm{L}$ & $18 \%$ \\
\hline $\begin{array}{l}\text { total injection } \\
\text { volume after } \\
\text { injection i } V_{\text {tot,i }}{ }^{\text {a }}\end{array}$ & $\begin{array}{l}\text { volume delivery } \\
\text { from syringe }\end{array}$ & $\begin{array}{l}\text { combining the } \\
\text { uncertainties of } \\
\text { individual } \\
\text { injection } \\
\text { volumes }\end{array}$ & $38.2 \mu \mathrm{L}$ & $0.764 \mu \mathrm{L}$ & $<1 \%$ \\
\hline
\end{tabular}

${ }^{\mathrm{a}}$ For the determination of the heat effects of individual injections $\Delta \mathrm{Q}(\mathrm{i})$, the uncertainty of components $V_{\text {tot }}$ (i-1) and $V_{\text {tot }}(\mathrm{i}-2)$ and $v_{\mathrm{i}}$ is done similarly and the uncertainty estimated as $2 \%$ of the respective volume.

\subsection{Determination of $\Delta H$ and $K$ Values and Their Uncertainty Estimation}

After the values and measurement uncertainties for each experimentally measured heat effect $\Delta Q(i)$ have been determined, a Python script was used for least squares curve fitting and the estimation of the resulting $\Delta H$ and $K$ values.

The fitting process works as follows: the experimental heat effects from individual injections, together with their determined measurement uncertainties are least-squares fitted to heat effects calculated using the theoretical binding model, to determine the $K$ and $\Delta H$ value for the studied reaction. The fitting is carried out in a Python script that uses the Nelder-Mead method [20] to determine the best estimates of $K$ and $\Delta H$ values and also estimates the uncertainty of the fit. The used Python script text and a more detailed explanation of its working process are presented in the SI.

Typically, two iterations of the script are sufficient - the first one uses peak area uncertainties determined from initial guesses for $K$ and $\Delta H$ values for the studied reaction, the second iteration uses peak area uncertainties calculated from the initial $K$ and $\Delta H$ estimates of the script. If the results do not change more than $1 \%$ between iterations, the second estimates are considered conclusive. When a notable difference occurred, the uncertainties were re-estimated for the new $K$ and $\Delta H$ estimates, the script rerun with the new uncertainty estimates and the difference of the results checked again, until the difference between values found between two consecutive iterations was below $1 \%$.

The current component by component approach carried out this far cannot investigate the uncertainty of data fitting between the theoretical and experimental titration curve. This estimation was done during the fit inside the Python script.

Four experiments were carried out, varying the concentration ratio of $\mathrm{K}^{+}$and $18 \mathrm{C} 6$. The results of experiments conducted in this study are displayed in Table 4 and in Figure 1.

The uncertainty estimate from the script cannot give information about the extent of contribution of the sources of measurement uncertainty towards the combined measurement uncertainty and in practice mainly estimates the goodness of the fit between computational and experimental data points (in the range of their estimates uncertainties). However, we can investigate whether the results obtained by script will fit inside the uncertainty limits of our final estimate. 
Darnell et al., J. Chem. Metrol. 15:1 (2021) 25-37

Table 4. Results of using different data fitting methods for experiments 1-4. The experiments were carried out in the time span of 2 months. The uncertainty estimates are the estimates of goodness-of-fit between the model and experimental points.

\section{Experiment no.}

\begin{tabular}{lcccccc}
\cline { 2 - 7 } & K1 & K2 & K3 & K4 & Average & SD \\
\hline $\mathrm{c}_{18 \mathrm{C} 6}(\mathrm{M})$ & 0.005 & 0.005 & 0.005 & 0.005 & & \\
$\mathrm{c}_{\mathrm{KCl}}(\mathrm{M})$ & 0.05 & 0.05 & 0.1 & 0.1 & & \\
“c $c$ " value & 0.575 & 0.575 & 0.575 & 0.575 & & \\
$K$ & 69.22 & 69.15 & 70.83 & 70.46 & 69.92 & 0.86 \\
$u(K)$ & 1.08 & 1.09 & 0.55 & 0.56 & & \\
$\Delta H(\mathrm{~kJ} / \mathrm{mol})$ & -27.64 & -27.14 & -25.78 & -25.87 & -26.61 & 0.92 \\
$u(\Delta H)(\mathrm{kJ} / \mathrm{mol})$ & 0.84 & 0.83 & 0.36 & 0.36 & & \\
\hline
\end{tabular}

SD: Standard deviation

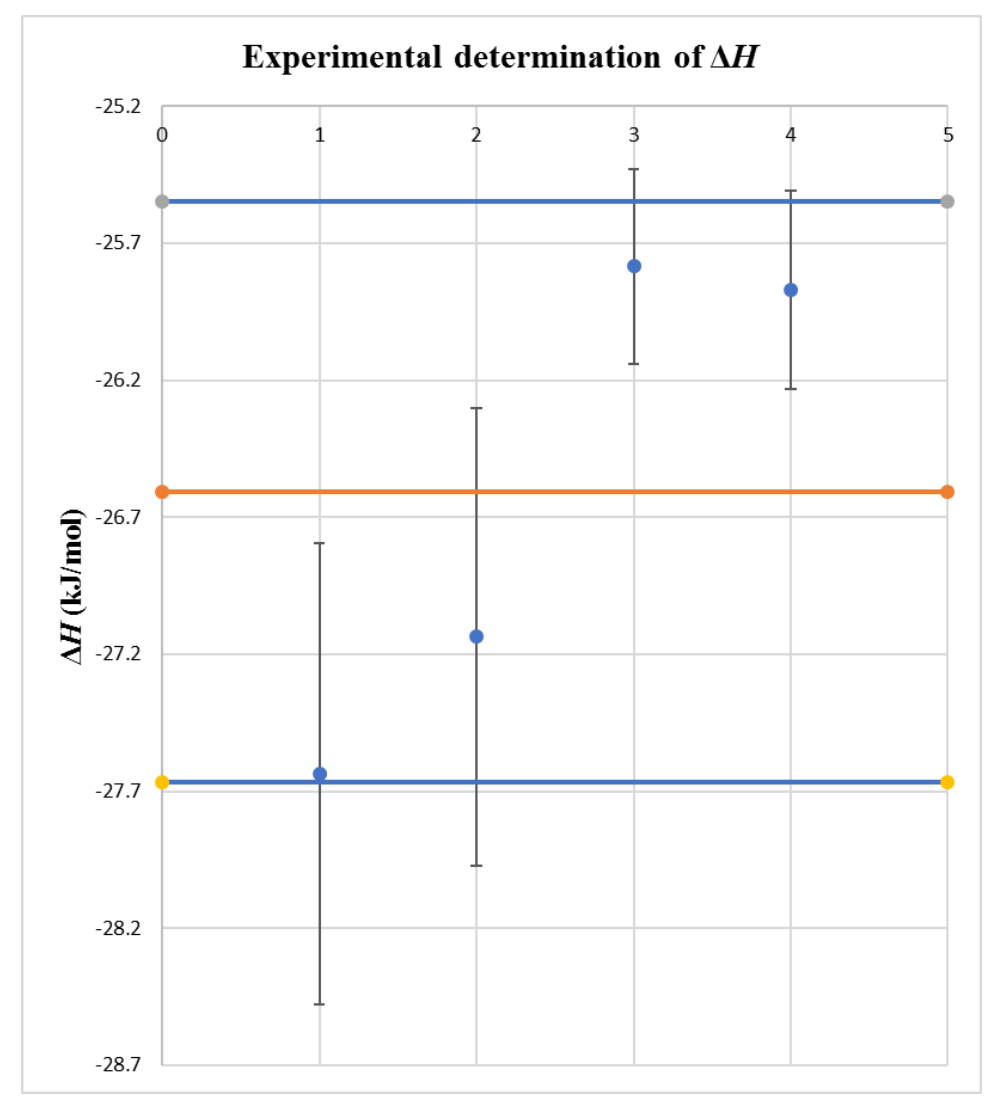

Figure 1. Experimentally determined $\Delta H$ values of individual experiments, the determined average value and the measurement uncertainty. The error bars represent the standard uncertainties of the $\Delta H$ values from the fitting procedure.

In order to do component-by-component uncertainty estimation of $\Delta H$, the dependence of $\Delta H$ from the total heat determined in the experiment $\left(Q_{\mathrm{tot}}\right)$, as well as all other experimental parameters is used as the model, expressed by equations (7), (3), (4) and (5). Equations (3)-(5) are used to calculate the values of active concentrations of the cation and the receptor $\left(X_{\mathrm{t}}\right.$ and $\left.M_{\mathrm{t}}\right)$ from the concentrations of the initial working solutions $\left(c_{s y r}\right.$ and $\left.M_{\mathrm{t} 0}\right)$. Equation (7) is derived from equation (2). The example file with uncertainty calculation using the Kragten approach can be seen is Supplementary Files. 


$$
\Delta H=\frac{2 Q}{n M_{\mathrm{t}} V_{0}\left[1+\frac{X_{\mathrm{t}}}{n M_{\mathrm{t}}}+\frac{1}{n K M_{\mathrm{t}}}-\sqrt{\left(1+\frac{X_{\mathrm{t}}}{n M_{\mathrm{t}}}+\frac{1}{n K M_{\mathrm{t}}}\right)^{2}-\frac{4 X_{\mathrm{t}}}{n M_{\mathrm{t}}}}\right]}
$$

After the application of component-by-component uncertainty estimation, the final reaction enthalpy is $\Delta H=-26.6 \mathrm{~kJ} / \mathrm{mol}$ with combined standard uncertainty $u_{\mathrm{c}}(\Delta H)=1.1 \mathrm{~kJ} / \mathrm{mol}$.

Figure 1 shows how well our individual experiments and their goodness-of-fit uncertainties compare with the combined standard uncertainty limits. It can be seen that nearly all results are inside the standard uncertainty limit and all results do fit inside this limit by the fitting uncertainties.

Table 5 shows the uncertainty budget for $\Delta H$. The most important components are the uncertainty of the measured total heat effect of the experiment $\left(Q_{\mathrm{tot}}\right)$ and the initial concentration of $18 \mathrm{C} 6$ in the cell $M_{\mathrm{t} 0}$.

Table 5. Uncertainty budget for the uncertainty of $\Delta H$.

\begin{tabular}{lcccc}
\hline \multirow{2}{*}{ Input quantities } & \multicolumn{4}{c}{ Uncertainty budget for $\boldsymbol{\Delta H}$} \\
\cline { 2 - 5 } & Example value & $\begin{array}{l}\text { Standard } \\
\text { uncertainty of } \\
\text { input value }\end{array}$ & $\begin{array}{l}\text { Uncertainty } \\
\text { components of } \\
\boldsymbol{\Delta} \boldsymbol{H}(\mathbf{k J} / \mathbf{m o l})\end{array}$ & $\begin{array}{l}\text { Contribution } \\
\text { to } \boldsymbol{\Delta} \boldsymbol{H} \\
\text { uncertainty }\end{array}$ \\
\hline total heat $Q_{\text {tot }}$ & $-7.54 \mathrm{~mJ}$ & $0.2 \mathrm{~mJ}$ & -0.7 & $40 \%$ \\
equilibrium constant $K$ & 69.9 & 2 & 0.0 & $17 \%$ \\
Active volume $V_{0}$ & $200.6 \mu \mathrm{L}$ & $4 \mu \mathrm{L}$ & 0.4 & $7 \%$ \\
stoichiometry coefficient $n$ & 1 & 0 & 0.3 & $0 \%$ \\
syringe concentration & $0.05 \mathrm{M}$ & $0.001 \mathrm{M}$ & 0.4 & $19 \%$ \\
$c_{\text {syra }}^{\mathrm{a}}$ & & & & $12 \%$ \\
initial cell concentration $M_{\mathrm{t} 0}{ }^{\mathrm{b}}$ & $0.005 \mathrm{M}$ & $0.0001 \mathrm{M}$ & 0.5 & $5 \%$ \\
total injection volume $V_{\text {tot }}{ }^{\mathrm{b}}$ & $38.2 \mu \mathrm{L}$ & $0.764 \mu \mathrm{L}$ & 0.2 & \\
\hline
\end{tabular}

a The parameter is used for the calculations of $X_{\mathrm{t}}$, according to equations (4) and (5).

${ }^{\mathrm{b}}$ The parameter is used for the calculation of $M_{\mathrm{t}}$, according to equation (3).

Comparing the results of this study with previously published literature values (Table 1), reveals that if we assume similar uncertainties for the other authors the $\Delta H$ values found in this work are in agreement with the majority of the literature $\Delta H$ values and those are in general in agreement with each other.

At the same time, the $\log K$ value in this study is $0.17-0.3 \log$ units lower than in literature. This could possibly be caused by the difficulties in accurate $K$ value determination from titration curves where the majority of the receptor has not been bound to complex by the end of the reaction. As our approach differs from the normally used Wiseman isotherm fitting, these experiments do not achieve a high rate of complexation at the end of the titration. The traditional approach advises for 50-90\% complexation, our experiments reach at most around 10\% complexation. It can be seen from our results that experiments of this type are unable to correctly determine the binding constant $K$ values.

The following effects were identified as potentially significant sources of measurement uncertainty. Some of them cannot be easily quantified on their own and should be considered when planning the experiments:

(1) Errors from the accuracy in data treatment for low-affinity systems. When the " $c$ " value of the experiment is low (" $c$ " values $<1$ ), the calorimetric titration curve is flat and may lack sufficiently distinguishable features for the standard data treatment to determine accurate $\Delta H$ and $K$ estimates. When experiments are planned, care should be taken to account for the low binding affinity to ensure that a sufficient part of the receptor can be bound into the complex at the end of the titration. With insufficient binding and low " $c$ " experiments, data treatment will be difficult and the measurement uncertainty increases notably.

(2) Uncertainty of the reaction stoichiometry coefficient $n$. Using the component-by-component approach allows checking how uncertainty contributions to the combined uncertainty change when the uncertainty of a single component increases or decreases. Investigating the effects of changes in input 
Darnell et al., J. Chem. Metrol. 15:1 (2021) 25-37

uncertainties confirms that knowing and fixing the value of the stoichiometry coefficient $n$ is necessary for calorimetric measurements under low $c$ conditions. Even a 3\% error in the value of $n$ would make it the largest uncertainty contributor; a $10 \%$ error in the estimate of $n$ value would cause an increase of the uncertainty estimate of $\Delta H$ by 2.3 times!

(3) Correctly accounting for heat of dilution. This effect occurs due to solvation of concentrated cation solution in the aqueous solution in the calorimeter cell. The magnitude of this effect depends on the properties of the host and guest, concentration of the solution and volumes of injection and titrant in cell. When different concentrations are used in replicate experiments, the heats of dilution will also be different and require new experiments to be accounted for correctly. This effect will be larger with higher titrant concentrations. At very high titrant concentrations, it is possible that the effect becomes too large to determine accurately due to instrumental limits. This was the reason the experiments in this study were not planned to $90 \%$ or a higher rate of receptor conversion.

(4) Instrument baseline correctness and stability. All experimental curves should be studied for baseline stability and lack of random errors (caused by vibration around instrument during the experiment, air bubbles in cell/syringe etc.) Calorimeters of this type are highly vibration sensitive [16], which can cause potential baseline errors during the measurement. It is important to check the stability of the baselines for all measurements, including those of the heat of dilution experiments. Data with large deflections in baselines may be unusable, so care should be taken that the experiments would not be disturbed by external vibration (nearby instruments in the laboratory, people walking in the laboratory constantly during the measurement, stability of the furniture the calorimeter is located on etc.) Direct evaluation of the uncertainty due to baseline irregularities or peak area reproducibility is difficult. A more practical approach is carrying out replicate measurements and evaluating the baseline effect (of course, in combination with numerous other effects) from the reproducibility of the results.

(5) Volume errors. It can be hard to determine the exact volume for filling the calorimeter cell and the exact active volume of the calorimeter during titration. The instrumental stirring during the experiment is not instant and is difficult to quantify/study. This can cause a concentration error which will in turn cause large misestimations during data treatment. Some previous studies have advised using a calibration reaction to estimate the active volume and volume delivery errors more accurately [23]. For stirring stability, it is important to ensure that the instrument`s syringe is not bent.

(6) Solution cross-contamination. When the instrument is not washed thoroughly enough (rinsed multiple times with studied solutions between measurements), even small leftovers of the previous solution can completely skew the estimated binding equilibrium. This can be avoided by doing "titration of water with water" reference measurements between titration experiments and looking for unexpected baseline shapes, notable heat effects from titration injections or moving baseline during the experiment. All these indicate cross contamination.

\section{Conclusions}

The binding between 18 -crown-6-ether and $\mathrm{K}^{+}$cation was studied with a small-volume isothermal titration calorimeter, at low $c$ values $(c<1)$. The thermodynamic parameters of this model reaction were determined, and the results compared with data from multiple literature sources.

A thorough uncertainty estimation was carried out using the ISO-GUM component-by component approach to estimate the measurement uncertainty of the measured heat effect obtained from each injection, these uncertainties were used in data fitting with a custom Python script to obtain the final estimate of uncertainty for the reaction enthalpy and binding constant values.

Investigation of the Kragten uncertainty budget revealed that the largest contributions to experimental uncertainty arise from errors in the experimental volumes - both the injection volume delivered during the titration with each injection and the accuracy of reactant concentrations, active volume and cell fill volume determination at the start of titration.

The model also allows to account for the effects of peak area integration stability (baseline stability) and the effects of errors in the subtraction of heat of dilution experiment. Testing the effect of increasing component uncertainty to the overall uncertainty estimate from the model shows that while with good 
experiment planning, these components have relatively small contributions to the overall uncertainty estimate, when instrumental or experimental problems occur (e.g. baseline deflections; crosscontamination; use of too high concentrations etc.), these components can become the largest contributors to the measurement uncertainty.

According to the results of this study, for low affinity systems, it is possible to obtain an estimate for $\Delta H$ value $-26.6 \mathrm{~kJ} / \mathrm{mol}$ with the combined measurement standard uncertainty of $1.1 \mathrm{~kJ} / \mathrm{mol}$. Comparing the results of this study with previously published literature values (Table 1), reveals that if we assume similar uncertainties for the other authors the $\Delta H$ values found in this work are in agreement with the majority of the literature $\Delta H$ values and those are in general in agreement with each other.

The results of this study indicate the accurate estimation of $K$ was not possible under our conditions and would require experimental planning where a higher complexation rate is achieved at the end of the experiment. If it is inevitable to work under low " $c$ " conditions, then it seems more practical to use $K$ estimates determined by a different method and only use microcalorimetry to determine $\Delta H$.

\section{Acknowledgements}

This research was funded by the Estonian Research Council grant PRG690 and by the EU through the European Regional Development Fund under project TK141 (2014-2020.4.01.15-0011). This study is part of the PhD thesis of Astrid Darnell. We would like to thank prof. T. Tenson`s group of the University of Tartu, Institute of Technology, whose Microcal iTC microcalorimeter was used for the experiments.

\section{Supporting Information}

Supporting information accompanies this paper on http://www.acgpubs.org/journal/ journal-ofchemical-metrology

\section{ORCID}

Author Name:

Astrid Darnell: 0000-0002-9719-2533

Lauri Sikk: 0000-0002-4015-1247

Ly Porosk: $0000-0002-4664-4560$

Ivo Leito: $\underline{0000-0002-3000-4964}$

\section{References}

[1] T. Wiseman, S. Williston, J.F. Brandts and L.-N. Lin (1989). Rapid measurement of binding constants and heats of binding using a new titration calorimeter, Anal. Biochem. 179, 131-137.

[2] L.D. Hansen, G.W. Fellingham and D.J. Russell (2011). Simultaneous determination of equilibrium constants and enthalpy changes by titration calorimetry: Methods, instruments, and uncertainties, Anal. Biochem. 409(2), 220-229.

[3] J. Tellinghuisen (2007). Optimizing experimental parameters in isothermal titration calorimetry: Variable volume procedures, J. Phys. Chem. B. 111 (39), 11531-11537.

[4] N.E. Grossoehme, A.M. Spuches and D.E. Wilcox (2010). Application of isothermal titration calorimetry in bioinorganic chemistry, J. Biol. Inorg. Chem. 15, 1183-1191.

[5] S.L. Tobey and E.V. Anslyn (2003). Studies into the Thermodynamic origin of negative cooperativity in ion-pairing molecular recognition, J. Am. Chem. Soc. 125 (36), 10963-10970.

[6] J. Tellinghuisen (2008). Isothermal titration calorimetry at very low c, Anal. Biochem. 373(2), 395-397.

[7] W.B. Turnbull and A.H. Daranas (2003). On the value of $c$ : Can low affinity systems be studied by isothermal titration calorimetry?, J. Am. Chem. Soc. 125 (48), 14859-14866.

[8] J. Tellinghuisen (2005). Optimizing experimental parameters in isothermal titration calorimetry, J. Phys. Chem. B. 109 (42), 20027-20035.

[9] R.M. Izatt, R.E. Terry, B.L. Haymore, L.D. Hansen, N.K. Dalley, A.G. Avondet, and J. J. Christensen (1976). Calorimetric titration study of the interaction of several uni- and bivalent cations with 15 -crown-5, 18-crown-6, and two isomers of dicyclohexo-18-crown-6 in aqueous solution at $25 .{ }^{\circ} \mathrm{C}$ and $\mu=0.1, \mathrm{~J}$. Am. Chem. Soc. 98, 7620-7626. 
Darnell et al., J. Chem. Metrol. 15:1 (2021) 25-37

[10] G. Michaux and J. Reisse (1982). Solution thermodynamic studies. Part 6. Enthalpy-entropy compensation for the complexation reactions of some crown ethers with alkaline cations: a quantitative interpretation of the complexing properties of 18-crown-6, J. Am. Chem. Soc. 104 (25), 6895-6899.

[11] Y. Liu and H. Jing (1987). A study on thermodynamic properties of crown ether complexation - effect of anions on the complexation of $1,4,7,10,13,16$-Hexaoxacyclooctadecan with some potassium salts in aqueous solution, Acta Physico Chim. Sinica 3, 11-15.

[12] K. Ozutsumi and S. Ishiguro (1992). A precise calorimetric study of 18-Crown-6 Complexes with sodium, potassium, rubidium, caesium, and ammonium ions in aqueous solution, Bull. Chem. Soc. Jpn. 65 (4), $1173-1175$.

[13] J.N. Spencer, J.E. Mihalick, T.J. Nicholson, P.A. Cortina, J.L. Rinehimer, J.E. Smith, X. Ke, Q. He and S. E. Daniels (1993). Comparison of the macrocyclic effect for ether hosts in aqueous and organic solvents, J. Phys. Chem. 97, 10509-10512.

[14] P. Wang, R.M. Izatt, S.E. Gillespie, J.L. Oscarson, X.X. Zhang, C. Wang, C. Wang and J. D. Lamb (1995). Thermodynamics of the interaction of 18 -crown- 6 with $\mathrm{K}^{+}, \mathrm{Ti}^{+}, \mathrm{Ba}^{2+}, \mathrm{Sr}^{2+}$ and $\mathrm{Pb}^{2+}$ from 323.15 to 398.15 K, J. Chem. Soc. Faraday Trans. 91 (23), 4207-4213.

[15] H.-J. Buschmann, R.-C. Mutihac and E. Schollmeyer (2008). Complex formation of 18-crown-6 with metal cations and ammonium ions in dioxane-water mixtures, Thermochim. Acta. 472 (1-2), 17-19.

[16] S.A. Kantonen, N.M. Henriksen and M.K. Gilson (2018). Accounting for apparent deviations between calorimetric and van't Hoff enthalpies, Biochim. Biophys. Acta Gen. Subj. 1862 (3), 692-704.

[17] S.A. Kantonen, N.M. Henriksen and M.K. Gilson (2017). Evaluation and minimization of uncertainty in ITC binding measurements: Heat error, concentration error, saturation, and stoichiometry, Biochim. Biophys. Acta Gen. Subj. 1861 (2), 485-498.

[18] H.J. Buschmann and E. Schollmeyer (1999). A test reaction from macrocyclic chemistry for calorimetric titrations, Thermochim. Acta. 333 (1), 49-53.

[19] J. Tellinghuisen (2018). Critique of methods for estimating heats in isothermal titration calorimetry, Anal. Biochem. 563 (15), 79-86.

[20] J.A. Nelder and R. Mead (1965). A simplex method for function minimization, Comput. J. 7, 308-313.

[21] S.L.R. Ellison, A. Williams, and Eurachem Working Group on Uncertainty in Chemical Measurement. Quantifying uncertainty in analytical measurement, Eurachem, London, 1995.

[22] MicrocalTM iTC200 System User Manual MAN0560, 2014.

[23] J. Tellinghuisen (2007). Calibration in isothermal titration calorimetry: Heat and cell volume from heat of dilution of $\mathrm{NaCl}(\mathrm{aq})$, Anal. Biochem. 360 (1), 47-55.

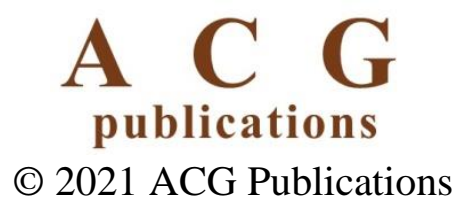

